\title{
Impact of Physical Inactivity on the Multifactorial Process of Developing Cancer-Related Cognitive Impairments
}

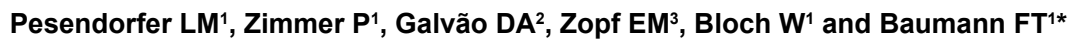

${ }^{1}$ Department of Molecular and Cellular Sport Medicine, German Sport University Cologne, Institute of Cardiovascular Research and Sport Medicine, Germany

${ }^{2}$ Exercise Medicine Research Institute, Edith Cowan University, Australia

${ }^{3}$ Institut for Health and Aging, Australien Catholic University, Melbourne Australia

\begin{abstract}
Many cancer survivors report cognitive impairment before, during and after medical treatment. It is well known that many factors other than chemotherapy take part in the development of these cancer- related cognitive impairments $(\mathrm{CRCl})$, such as cancer-related fatigue, psychological as well as molecular factors. The purpose of this review is to describe the impact of physical inactivity in the genesis of these cognitive changes in cancer patients. References for this narrative review comes primarily from PubMed, Cochrane Library and Livivo databases. It contains literature search from October 2003 to April 2016. Our search was focused on available data for the influence of physical inactivity on cognitive performance of cancer patients. Cancer patients have decreased levels of physical activity during treatment. It is suggested that lack of physical activity in general is associated with cognitive decline. At present, most available data comes from trials with heart failure patients as well as results from bed rest studies in space medicine. The development of $\mathrm{CRCl}$ is a multifactorial process with physical inactivity appearing to play an important role. However, the available data remains limited and future trials are needed to examine the specific role of physical inactivity on CRCI.
\end{abstract}

Keywords: Bed rest; Cancer-related cognitive impairments; Chemobrain; Cognition; Immobilization; Lack of exercise; Physical inactivity

\section{Introduction}

In 2012, about 14 million new cases of cancer were reported worldwide [1]. Due to a variety of new therapies, especially targeted treatment approaches, survival has improved substantially in recent years [2]. However, cancer itself and its medical treatment are accompanied by a number of treatment-related adverse effects and toxicities including detrimental effects on physical, psychological and social aspects [3]. An important and clinical side-effect has often been termed as "chemobrain" [3], which describes cognitive dysfunction associated to cancer treatment [4,5]. This broad term includes a variety of cognitive impairments that may occur either temporarily or persist as a long-term consequence of treatment $[3,6-8]$. Cognitive dysfunction mainly relates to memory, attention, executive functions, learning and speed of information processing decline [4,5,9]. Further, it is also known that other factors than cancer treatments influence the development of cognitive changes in cancer patients suggesting that this is a multifactorial process $[3,10]$. As a result, experts have proposed the term "cancer-related cognitive impairments" (CRCI) which we will use hereinafter [11].

It has been suggested the percentage of patient suffering from CRCI varies between $16 \%$ to $75 \%$ depending on type of treatment (e.g. chemotherapy, radiation) and time point of measurement (before, during or after medical treatment) [3], also affecting patients 'quality of life [12].

The purpose of this narrative review is to present a descriptive overview of the multifactorial process of CRCI in cancer patients and the impact of physical inactivity during this process. Numerous studies have reviewed the effects of physical activity on health; however, few studies are available on physical inactivity or sedentary behavior [13].

Physical activity appears to decrease from time of cancer diagnosis to initiation of anticancer treatment with patients showing the lowest levels during treatment with a subsequent increase following therapy
[14-16]. The role of physical inactivity in the multifactorial process of CRCI development has not been extensively described and is the focus of our review (Figure 1).

\section{Materials and Methods}

This narrative review has been divided into four sections: 1) multifactorial aspects generating CRCI, 2) the role of physical inactivity, 3) discussion, and 4) conclusion. Information from this narrative review was obtained by searching the PubMed, Livivo and Cochrane Library databases from October 2003 to June 2016. A first search focuses on the influence of physical inactivity on development of cognitive impairments in cancer patients. Therefore, a combination of following terms was used: on the one hand "cancer-related cognitive decline", "cancer-related cognitive dysfunctions", "cancer-related cognitive impairments", "chemobrain", "chemofog", "post-chemotherapy cognitive impairment", "cancer brain", "cancer associated cognitive impairment", "cancer-therapy-associated cognitive impairment" and on the other hand "bed rest", "immobilization", "lack of exercise", "physical inactivity". There were no publications referring to this aspect exclusively. As such, a second search was conducted focusing on influence of physical inactivity on cognitive performance in general. Therefore, a combination of the following terms was used: "physical inactivity", "bed rest", "immobilization", "lack of exercise" and on the

*Corresponding author: Baumann FT, Department of Molecular and Cellular Sport Medicine, German Sport University Cologne, Institute of Cardiovascular Research and Sport Medicine, Am Sport park Müngersdorf 650933 Köln Germany, Tel: 02214982 4821; Fax: 02214982 8370; E-mail: f.baumann@dshs-koeln.de

Received July 04, 2016; Accepted August 29, 2016; Published August 31, 2016

Citation: Pesendorfer LM, Zimmer P, Galvão DA, Bloch W, Baumann FT (2016) Impact of Physical Inactivity on the Multifactorial Process of Developing CancerRelated Cognitive Impairments. J Cancer Sci Ther 8: 222-227. doi: 10.4172/19485956.1000417

Copyright: () 2016 Pesendorfer LM, et al. This is an open-access article distributed under the terms of the Creative Commons Attribution License, which permits unrestricted use, distribution, and reproduction in any medium, provided the original author and source are credited. 


\section{Impact of Physical Inactivity on \\ "Cancer Related Cognitive Impairments"}

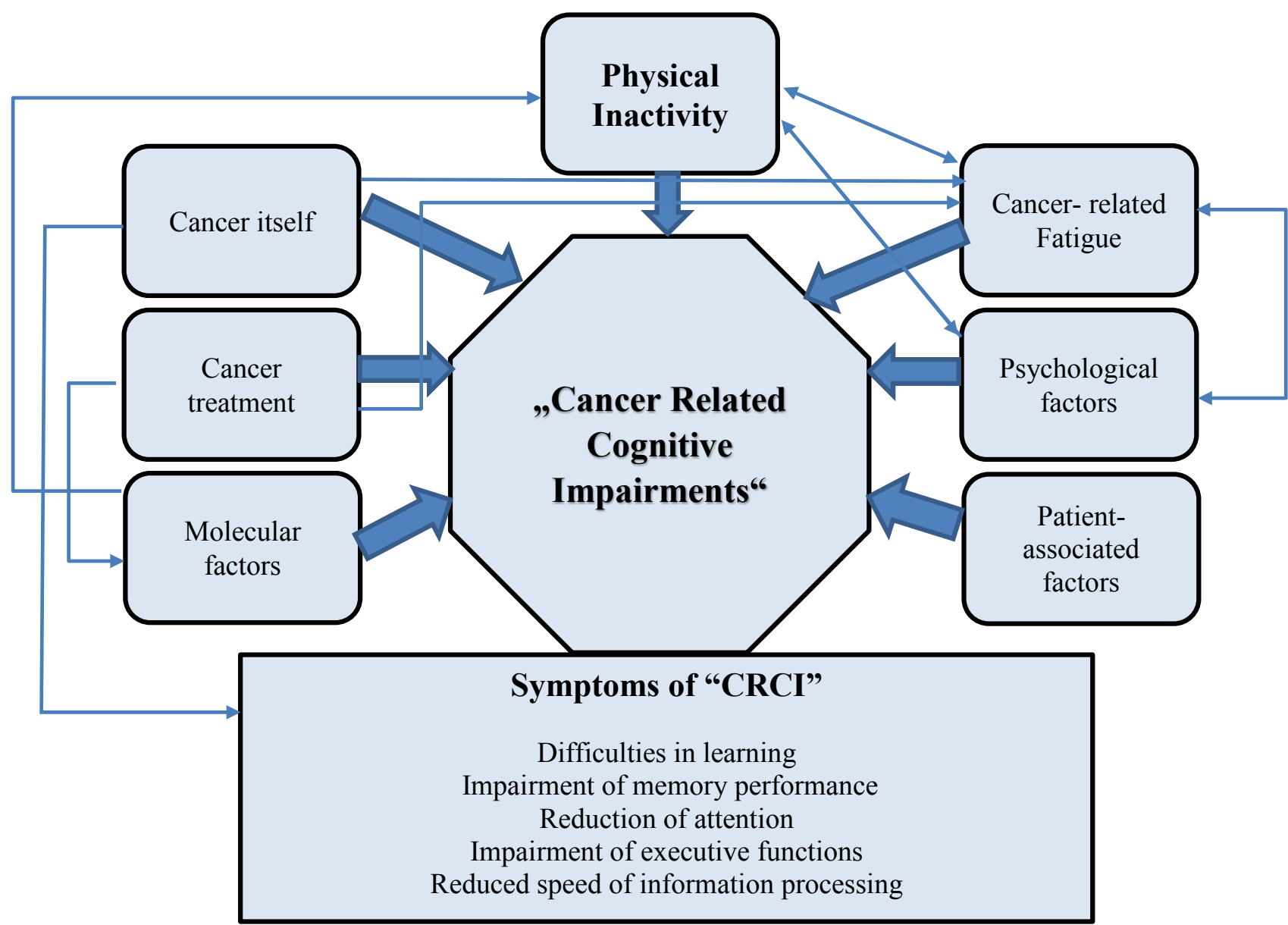

Figure 1: Impact of physical inactivity on "Cancer Related Cognitive Impairments (CRCI)" in a multifactorial genesis.

other hand "cognition" "cognitive decline", "memory", "attention", "learning", "executive functions". As a result, six relevant articles were found in total. For background information concerning development or treatment of CRCI we also used information from Google Scholar database. Finally, data from world cancer report 2014 and American Association for Cancer Research were included in this review [1].

\section{Multifactorial Aspects Generating CRCI}

In past years, CRCI was placed at the center of numerous clinical trials (e.g. by Hermelink et al. [18], Lepage et al. [19], Nudelman et al. [20], Wefel et al. [7] and Whitney et al. [8]), particularly in patients with breast cancer, and also in some rodent-based research (e.g. by Briones et al. [4], Han et al. [21] and Seigers et al. [22-24]).

\section{Chemotherapeutics}

Several studies have shown that chemotherapeutics negatively impacts on a variety of ways the central nervous system functions. For example, cytostatic agents such as cyclophosphamide, 5-flouracil (5FU), doxorubicin and methotrexate (MTX) have shown to decrease neurogenesis and/or cell proliferation in the hippocampus. These changes might be associated with impaired memory performance and difficulties in learning [4,5,22-27]. In rodent research, it is also reported that cytostatic agents (e.g. carmustine, cytarabine, cisplatin, 5-FU, MTX) affect the oligodendrocytes which may lead to damage of the white matter $[21,24,26-28]$ hence potentially reducing speed of information processing [27]. Furthermore, cytostatic therapy induces reduction of the gray matter in certain brain areas, such as the cerebellum and the prefrontal cortex. Loss of gray matter density seems to be associated with impairment in executive functions, verbal memory und working memory $[9,19,29]$.

Several anti-tumor therapies also appear to lead to alterations in cerebral blood flow. For example, some medical treatments induce cerebral hyperperfusion accompanied by impairment in neuropsychological test performance. In contrary, a decrease of gray matter density in those brain areas is associated with cerebral hypoperfusion [20].

\section{Hormone therapy}

CRCI may even occur after hormone therapy (e.g. tamoxifen, aromatase inhibitors, androgen deprivation therapy) $[3,9,10]$ 
Consequently, androgen deprivation therapy seems to be associated with a reduced response time considering to cognitive function [3]. Tamoxifen is an estrogen receptor antagonist and its use is accompanied by impaired cognitive capabilities [3]. Aromatase inhibitors intervene in the estrogen metabolism by blocking the conversion of androgens to estrogens resulting in reduced levels of estrogen and leading to cognitive impairments [3].

\section{Radiation therapy}

Research undertaken with animal models has suggested that cranial radiation decreases neurogenesis in the hippocampus which leads to impaired hippocampal memory functions [30]. It has been suggested that irradiation doses of a least 5 Gray (Gy) are sufficient to induce these changes [30]. Furthermore, it is assumed that radiation-induced neuroinflammation is associated with hippocampal dysfunction [30]. Among other factors oxidative stress and proinflammatory cytokines such as tumor necrosis factor $\alpha$ (TNF- $\alpha)$ and interleukin-1 $\beta$ (IL-1 $\beta$ ) are involved in these inflammatory processes leading to neuronal impairments (e.g. through affecting mitochondrial functions). As a result, survival and differentiation of neural progenitor cells can be negatively affected. Finally, decrease of hippocampal neurogenesis is also induced [30].

Brain- derived neurotrophic factor (BDNF) is an important modulator in the context of the proliferation, differentiation and survival of cerebral neurons. Moreover, it seems to take a significant role in hippocampus dependent learning and memory processes. Cerebral irradiation leads to a decrease of the BDNF levels in the serum associated with disruption of these processes [30]. Furthermore, data from animal research suggests an association between low levels of $\mathrm{BDNF}$ and the emergence of depressive symptoms [30].

\section{Molecular underspinning mechanisms}

On a molecular level some inflammatory cytokines such as IL-1, IL-6 and TNF- $\alpha$ seem to take part on the genesis of CRCI [2]. It is well documented that an increased BMI which can be driven by a sedentary lifestyle contributes to elevated PR inflammatory cytokine levels. A prospective cohort study with breast cancer patients staged I-III have shown impairment of speed performance in patients with increased serum levels of IL- $1 \beta$ and poorer verbal memory in patients with increased levels of TNF- $\alpha$. An increased level of IL- 6 seems to favor a rise of self- perceived cognitive impairment. In contrast, higher serum levels of IL- 4 were associated with improvement in speed performance [31]. A positive relationship has been reported between higher serum levels of IL- 6 and an increase of cancer- related fatigue [32]. Increased levels of several inflammatory cytokines appear to be a consequence of both cancer itself and anticancer treatment $[3,31]$.

Furthermore, it is suspected that the tumor itself may negatively affect cognitive function in different ways [10,27]. Among other aspects, it is suggested that presence of cancer is accompanied by reduced levels of BDNF. There might also be an association with damaged DNA and damaged repair mechanisms of DNA [27,33].

As mentioned above, studies investigating the relationship between physical inactivity and CRCI are sparse. However, exercise intervention studies in both, humans and animals suggest that physical activity induces an anti-inflammatory cytokine profile, e.g. by increasing the number of IL-10 releasing regulatory T-cells and by reducing fat mass [34,35]. Additionally, physical activity induces a number of neuroprotective and neurotrophic factors such as BDNF, VEGF, IGF-1 and 5-HT [36]. Interestingly, such increase impact both peripheral and central concentrations of the named factors [37].

\section{Patient-associated factors}

It is also proposed that there are patient-associated factors like genetic predisposition or changes in hormonal status (e.g. decreased levels of neuroprotective estrogen) which favor the development of CRCI [3,29]. Among several aspects, risk for developing CRCI seems to be associated with genotype of catechol o- methyltransferase (COMT) thereby where the gene coding for this enzyme is located on chromosome 22q11. At codon 158 either valine and / or methionine are incorporated. Depending on the installed amino acid, COMT is modified in its function by the breakdown of dopamine in the prefrontal cortex. If valine is located on this place degradation takes $3-4 \mathrm{x}$. As a result, CRCI appears to be more pronounced in valine carriers than in methionine carriers [10,38,39]. Moreover, cancer patients with the allele e4 of the apolipoproteine E (ApoEe4) have an increased risk in developing CRCI. For example, Koleck et al. have shown in a prospective, longitudinal study that cancer patients with ApoEe4 have worse results in verbal learning and memory than controls [39]. ApoEe4 is also often present in patients suffering from Alzheimer's disease $[3,29]$.

\section{Psychological factors}

CRCI can be divided into self- perceived and objective-measured cognitive impairments. They may not necessarily have to appear together in cancer patients suffering from cognitive changes [18]. Subjectively perceived cognitive impairments correlates with fatigue, extent of depression and level of negative attitude of the patient $[3,18]$. Interestingly, in some patients CRCI is present before the initiation of anticancer treatment [40,41]. Wefel et al. suggested an association between level of patient's distress and this phenomenon [40]. Data on the prospective "Cognicare study" by Hermelink et al. also indicated cancer-related posttraumatic stress as an important causal factor for pretreatment cognitive decline [41].

\section{Lifestyle Factors}

Severity of CRCI is likely to also be dependent on lifestyle factors. Hartmann et al. described decreases of cognitive performance to be associated with sleep deprivation in women with breast cancer. Moreover, higher levels of physical activity appear to prevent CRCI [42].

\section{Physical inactivity and CRCI}

Several clinical trials with cancer patients have shown that physical activity decreases after diagnosis [14-16,43,44]. For instance, physical inactivity is associated with a decrease of hippocampal neurogenesis and loss of hippocampal volume, and this may result in cognitive impairment [45]. This leads to memory impairment and problems in decision-making. Inactivity is also associated with a decreased neurogenesis in the cerebellum, inducing problems in motor programs, and in the olfactory bulb [45]. Alosco et al. described a loss of gray matter volume and cortical thickness in patients with poor physical fitness in patients with heart failure. Importantly, the authors have suggested that such changes were not caused by cardiac disease or any other medical conditions [46]. In a longitudinal trial with 65 heart failure patients, Alosco et al. concluded that physical inactivity is closely associated with cerebral hypoperfusion. Reduced blood flow seems to have a negative effect especially on executive functions and attention [47]. Lipnicki et al. has also found a negative effect on executive functions and a delayed reaction time by 60 days of head-down bed rest [48]. Similarly, Liu et al. provided similar conclusions in their head-down bed rest- experiments [49]. However, results from bed rest studies concerning the influence on cognitive performance are inconsist with some studies reporting an 
improvement in cognitive performance, others reporting no significant effect and some reporting a detrimental effect [50]. These findings were independent of bed rest position and have been found in different types of cognition tests [50].

As described above, similarities between developing CRCI and the impact of physical inactivity on cognitive performance in general exist. Both lack of exercise and cancer treatment lead to alterations in cerebral blood flow associated with impairment in attention and executive function $[20,47]$. Further, they both induce partly comparable changes in brain structures like loss of gray matter in some brain regions $[9,19,29,46]$. Moreover, both lack of exercise and several anticancer treatments (chemotherapeutic agents, radiation) lead to a decreased hippocampal neurogenesis and hippocampal volume $[29,46]$. These changes are associated with memory impairment and problems in decision making $[4,5,22-25,27,45]$.

Exacerbation of fatigue, depression and anxiety favor the development of self- reported cognitive impairments in cancer patients $[3,6,18]$. Lack of exercise is also associated with an increased risk for fatigue and depression [51], which may consequently reduce the ability to exercise [52]. Further, data from clinical trials concerning the effect of physical activity on CRCI shows an improvement of self- reported cognitive impairment $[53,54]$. There might also be a positive effect on verbal memory, attention and function of frontal brain $[55,56]$. Moreover, levels of IL- $1 \beta$, IL- 6 and TNF- $\alpha$ are lower in patients engaging in regular exercise [54,57].

\section{Discussion}

The development of CRCI is a multifactorial process. In addition to anticancer treatment numerous other factors such as depression, anxiety, patient-associated factors, molecular factors, tumor itself and cancer-related fatigue takes part in this process $[3,10,27]$. As a result of the above mentioned communalities between CRCI's genesis and influence of physical inactivity on cognitive performance in general, we also suggest that physical inactivity is another important causative factor of CRCI. However, data concerning influence of physical inactivity on cognitive performance are limited and are primarily from studies in patients with heart failure or from bed rest studies in space medicine [46-50].

Despite numerous clinical trials and some rodent research in the last years, the phenomenon of CRCI is not yet fully understood. Current data suggests that especially anthracycline- based chemotherapeuticregimes have a negative effect on verbal memory performance and left precuneus connectivity [58]. Furthermore, the exact influence of hormone therapies is still unknown and therefore their exact role remains to be determined [3]. In addition, knowledge about the side effects of antibody therapy on cognitive impairment is very weak [59]. Moreover, currently data on the influence of psychological factors on development of CRCI differs among studies $[3,7,18]$.

Due to the large number of potential causative factors different treatment strategies exists to address CRCI. Time from active treatment seems to be one of the most important factors in the regression of CRCI as most patients' cognitive performance improves over the time [60]. Pharmacological treatment options are available but unfortunately are not well supported by clinical data $[3,61]$. Psychological intervention can also take place in treatment of mood disorders and cancerrelated fatigue $[62,63]$. In addition, other interventions such as skillfocused cognitive training seem to improve executive functions, memory functions, and speed of information processing [64]. Physical exercise intervention studies have also shown good efficacy in the management of CRCI [11,54-57,64,65]. Amongst several positive effects, improvement of verbal memory, attention and prefrontal lobe functions have been reported $[55,56]$. Physical exercise seems to also decrease level of proinflammatory cytokines like IL-6, IL-1 $\beta$ and TNF- $\alpha$ as well as levels of inflammation in general in humans [52,57]. Moreover, physical exercise leads to improvement in self- perceived cognitive impairment and quality of life $[53,54,57]$. Although drawing similar conclusions, results from these studies must be critically interpreted due to methodological limitations and small sample sizes. Further randomized controlled studies with larger sample sizes are required to confirm and expand current preliminary findings.

\section{Conclusion}

The development of CRCI is a multifactorial process. In this narrative review, we provided the multifactorial genesis of CRCI and particularly the role of physical inactivity in this context. Existing data on changes of physical activity levels in cancer patients and its associated effects on CRCI remains limited. Future trials are encouraged to focus on these aspects to confirm and expand current findings. Importantly, the influence of physical inactivity on development of CRCI needs to be determined via randomized- controlled trials.

\section{References}

1. Stewart BW, Wild CP (2014) World cancer report 2014, International Agency for Research on Cancer (I A R C), United Nations.

2. American Association for Cancer Research: AACR Cancer Progress Report 2014. Clin Cancer Res 20:1-112.

3. Argyriou AA, Assimakopoulos K, Iconomou G, Giannakopoulou F, Kalofonos HP (2011) Either called "chemobrain" or "chemofog," the long-term chemotherapyinduced cognitive decline in cancer survivors is real. J Pain Symptom Manage 41: 126-139.

4. Briones TL, Woods $\mathrm{J}$ (2011) Chemotherapy-induced cognitive impairment is associated with decreases in cell proliferation and histone modifications. BMC Neurosci 12: 124.

5. Nokia MS, Anderson ML, Shors TJ (2012) Chemotherapy disrupts learning neurogenesis and theta activity in the adult brain. Eur J Neurosci 36: 3521 3530 .

6. Mehnert A, Scherwath A, Schirmer L, Schleimer B, Petersen C, et al. (2007) The association between neuropsychological impairment, self-perceived cognitive deficits, fatigue and health related quality of life in breast cancer survivors following standard adjuvant versus high-dose chemotherapy. Patient Education and Counseling 66: 108-118.

7. Wefel JS, Lenzi R, Theriault R, Davis R., Meyers CA (2004) The Cognitive Sequelae of standard- Dose Adjuvant Chemotherapy in Women with Breast Carcinoma. Results of a Prospective, Randomized, Longitudinal Trial. CANCER 100: 2292-2299.

8. Whitney KA, Lysaker PH, Steiner AR, Hook JN, Estes DD, et al. (2008) Is"chemobrain" a transient state? A prospective pilot study among persons with non-small cell lung cancer. J Support Oncol 6: 313-321.

9. McDonald BC, Conroy SK, Ahles TA, West JD, Saykin AJ (2010) Gray matter reduction associated with systemic chemotherapy for breast cancer: a prospective MRI study. Breast Cancer Res Treat 123: 819-828.

10. Rick O (2014) Kognitive Dysfunktion oder Chemobrain. GMS Onkol Rehabil Sozialmed, 3.Doc04

11. Zimmer P, Baumann FT, Oberste M, Wright P, Garthe A, et al. (2016) Effects of Exercise Interventions and Physical Activity Behavior on Cancer Related Cognitive Impairments: A Systematic Review. Biomed Res Int 2016: 1820954.

12. Scherling CS, Smith A (2013) Opening up the window into "chemobrain": a neuroimaging review. Sensors (Basel) 13: 3169-3203.

13. Lee IM, Shiroma EJ, Lobelo F, Puska P, Blair SN, et al. (2012) Effect of physical inactivity on major non-communicable diseases worldwide: an analysis of burden of disease and life expectancy. Lancet 380: 219-229. 
Citation: Pesendorfer LM, Zimmer P, Galvão DA, Bloch W, Baumann FT (2016) Impact of Physical Inactivity on the Multifactorial Process of Developing Cancer-Related Cognitive Impairments. J Cancer Sci Ther 8: 222-227. doi: 10.4172/1948-5956.1000417

14. Howard-Anderson J, Ganz PA, Bower JE, Stanton AL (2012) Quality of life, fertility concerns, and behavioral health outcomes in younger breast cance survivors: a systematic review. J Natl Cancer Inst 104: 386-405.

15. Irwin ML, Crumley D, McTiernan A, Bernstein L, Baumgartner R, et al. (2003) Physical activity levels before and after a diagnosis of breast carcinoma: the Health, Eating, Activity, and Lifestyle (HEAL) study. Cancer 97: 1746-1757.

16. Lahart IM, Metsios GS, Nevill AM, Kitas GD, et al. (2016) Randomised controlled trial of a home-based physical activity intervention in breast cancer survivors. BMC Cancer 16: 234

17. Lahart IM, Metsios GS, Nevill AM, Carmichael AR (2014) Physical activity levels in women attending breast screening, receiving chemotherapy and post-breast cancer treatment; a cross-sectional study. Int J Environ Res Public Health 11: 5487-5496.

18. Hermelink K, Küchenhoff H, Untch M, Bauerfeind I, Lux MP, et al. (2010) Two different sides of "chemobrain": determinants and nondeterminants of selfperceived cognitive dysfunction in a prospective, randomized, multicenter study. Psycho- Onkology, 19.1321-1328.

19. Lepage C, Smith AM, Moreau J, Barlow- Krelina E, Wallis N, et al. (2014) A prospective study of gray matter and cognitive function alterations in chemotherapy- treated breast cancer patients. SpringerPlus 3: 44

20. Nudelman KNH, Wang Y, McDonald BC, Conroy SK, Smith DJ, et al. (2014) Altered Cerebral Blood Flow One Month After Systemic Chemotherapy for Breast Cancer: A Prospective Study Using Pulsed Aterial Spin Labeling MR Perfusion. PLoS ONE, 9: e96713.

21. Han R, Yang Y, Dietrich J, Luebke A, Mayer-Pröschel M, et al. (2008) Systemic 5 -fluorouracil treatment causes a syndrome of delayed myelin destruction in the central nervous system. Journal of biology 7: 12 .

22. Seigers R, Pourtau L, Schagen SB, van Dam FS, Koolhaas JM, et al. (2010) Inhibition of hippocampal cell proliferation by methotrexate in rats is not potentiated by the presence of a tumor. Brain Res Bull 81: 472-476.

23. Seigers R, Schagen SB, Beerling W, Boogerd W, van Tellingen O, et al (2008) Long-lasting suppression of hippocampal cell proliferation and impaired cognitive performance by methotrexate in the rat. Behav Brain Res 186: 168 175 .

24. Seigers R, Schagen SB, Coppens CM, van der Most PJ, van Dam FS, et al. (2009) Methotrexate decreases hippocampal cell proliferation and induces memory deficits in rats. Behav Brain Res 201: 279-284

25. Christie LA, Acharya MM, Parihar VK, Nguyen A, Martirosian V, et al. (2012) Impaired Cognitive Function and Hippocampal Neurogenesis following Cancer Chemotherapy. Clin Cancer Res 18: 1954-1965.

26. Monje M, Dietrich J (2012) Cognitive side effects of cancer therapy demonstrate a functional role for adult neurogenesis. Behav Brain Res 227: 376-379.

27. Seigers R, Fardell JE (2011) Neurobiological basis of chemotherapy-induced cognitive impairment: a review of rodent research. Neurosci Biobehav Rev 35 729-741.

28. Dietrich J, Han R, Yang Y, Mayer-Pröschel M, Noble M (2006) CNS progenitor cells and oligodendrocytes are targets of chemotherapeutic agents in vitro and in vivo. J Biol 5: 22

29. McDonald BC, Conroy SK, Smith DJ, West JD, Saykin AJ, et al. (2013) Frontal gray matter reduction after breast cancer chemotherapy and association with executive symptoms: a replication and extension study. Brain, Behaviour, and Immunity, 30: S117-S125.

30. Son Y, Yang M, Wang H, Moon C (2015) Hippocampal dysfunction caused by cranial irradiation. A review of the experimental evidence. Brain, Behaviour, and Immunity, 45: 287-296.

31. Cheung YT, Ng T, Shwe M, Ho HK, Foo KM, et al. (2015) Association of proinflammatory cytokines and chemotherapy- associated cognitive impairment in breast cancer patients: a multi- centered, prospective cohort study. Ann Oncol 26: 1446-1451

32. Saligan LN, Kim HS (2012) A systematic review of the association between immunogenomic markers and cancer- related fatigue. Brain Behav Immun 26: 830-848.

33. Mandelblatt JS, Hurria A, McDonald BC, Saykin AJ, Stern RA, et al. (2013) Cognitive effects of cancer and its treatments at the intersection of aging: what do we know; what do we need to know?. Semin Oncol 40: 709-725.
34. Wefel JS, Kesler R, Noll KR, Schagen SB (2015) Clinical Characteristics, Pathophysiology, and Management of Noncentral Nervous System CancerRelated Cognitive Impairment in Adult. CA Cancer J Clin, 65: 123-138.

35. Weinhold M, Shimabukuro-Vornhagen A, Franke A, Theurich S, Wahl P, et al. (2016) Physical exercise modulates the homeostasis of human regulatory $T$ cells. J Allergy Clin Immunol 137: 1607-1610.

36. Joshi RK, Lee SA (2014) Obesity related adipokines and colorectal cancer: a review and meta-analysis. Asian Pac J Cancer Prev 15: 397-405.

37. Papenberg G, Ferencz B, Mangialasche F, Mecocci P, Cecchetti R, et al. (2016) Physical activity and inflammation: effects on gray-matter volume and cognitive decline in aging. Hum Brain Mapp 37: 3462-3473.

38. Small BJ, Rawson KS, Walsh E, Jim HS, Hughes TF, et al. (2011) CatecholO-methyltransferase genotype modulates cancer treatment-related cognitive deficits in breast cancer survivors. Cancer 117: 1369-1376.

39. Koleck TA, Bender CM, Sereika SM, Ahrendt G, Jankowitz RC, et al. (2014) Apolipoprotein $\mathrm{E}$ genotype and cognitive function in postmenopausal women with early-stage breast cancer. Oncol Nurs Forum 41: E313-325.

40. Wefel JS, Lenzi R, Theriault R, Buzdar AU, Cruickshank S, et al. (2004) 'Chemobrain' in breast carcinoma?: a prologue. Cancer 101: 466-475.

41. Hermelink K, Voigt V, Kaste J, Neufeld F, Wuerstlein R, et al. (2015) Elucidating Pretreatment Cognitive Impairment in Breast Cancer Patients: The Impact of Cancer- Related Post- Traumatic Stress. J Natl Cancer Inst 107: 126.

42. Hartman SJ, Marinac CR, Natarajan L, Patterson RE (2015) Lifestyle factors associated with cognitive functioning in breast cancer survivors. Psychooncology 24: 669-675.

43. Blanchard CM, Denniston MM, Baker F, Ainsworth SR, Courneya KS, et al. (2003) Do adults change their lifestyle behaviors after a cancer diagnosis?. Am J Health Behav 27: 246-256.

44. Satia JA, Campbell MK, Galanko JA, James A, Carr C, et al. (2004) Longitudina changes in lifestyle behaviors and health status in colon cancer survivors. Cancer Epidemiol Biomarkers Prev 13: 1022-1031.

45. Pruimboom $L$ (2011) Physical inactivity is a disease synonymous for a nonpermissive brain disorder. Med Hypotheses 77: 708-713.

46. Alosco ML, Brickman AM, Spitznagel MB, Griffith EY, Narkhede A, et al. (2013) Poorer fitness level is associated with reduced structural brain integrity in heart failure. J Neurol Sci 328: 51-57.

47. Alosco ML, Spitznagel MB, Cohen R, Raz N, Sweet LH, et al. (2014) Decreased physical activity predicts cognitive dysfunction and reduced cerebral blood flow in heart failure. J Neurol Sci 339: 169-175.

48. Lipnicki DM, Gunga HC, Belavý DL, Felsenberg D (2009) Bed rest and cognition: effects on executive functioning and reaction time. Aviat Space Environ Med 80: 1018-1024.

49. Liu Q, Zhou R, Chen S, Tan C (2012) Effects of head-down bed rest on the executive functions and emotional response. PLoS One 7: e52160.

50. Lipnicki DM, Gunga HC (2009) Physical inactivity and cognitive functioning results from bed rest studies. Eur J Appl Physiol 105: 27-35.

51. Hayes SC, Spence RR, Galvão DA, Newton RU (2009) Australian Association for Exercise and Sport Science position stand: optimising cancer outcomes through exercise. J Sci Med Sport 12: 428-434.

52. Lucía A, Earnest C, Pérez M (2003) Cancer-related fatigue: can exercise physiology assist oncologists? Lancet Oncol 4: 616-625.

53. Reid-Arndt SA, Matsuda S, Cox CR (2012) Tai Chi effects on neuropsychological, emotional, and physical functioning following cancer treatment: a pilot study. Complementary Therapies in Clinical Practice, 18: 26-30.

54. Derry HM, Jaremka LM, Bennett JM, Peng J, Andridge R, et al. (2014) Yoga and self- reported cognitive problems in breast cancer survivors: a randomized controlled trial. Psycho- Oncology 24: 958-966.

55. Baumann FT, Drosselmeyer N, Leskaroski A, Knicker A, Krakowski- Roosen, et al. (2011) 12- Week Resistance Training with Breast Cancer Patients during chemotherapy: Effects on Cognitive Abilities. BreastCare 6: 142-143.

56. Miki E, Kataoka T, Okamura H (2014) Feasibility and efficacy of speed-feedback therapy with a bicycle ergometer on cognitive function in elderly cancer patients in Japan. Psychooncology 23: 906-913. 
Citation: Pesendorfer LM, Zimmer P, Galvão DA, Bloch W, Baumann FT (2016) Impact of Physical Inactivity on the Multifactorial Process of Developing Cancer-Related Cognitive Impairments. J Cancer Sci Ther 8: 222-227. doi: 10.4172/1948-5956.1000417

57. Oh B, Butow PN, Mullan BA, Clarke SJ, Beale PJ, et al. (2012) Effect of medical Qigong on cognitive function, quality of life, and a biomarker of inflammation in cancer patients: a randomized controlled trial. Supportive Care in Cancer 20: $1235-1242$.

58. Kesler SR, Blayney DW (2016) Neurotoxic Effects of Anthracycline- vs Nonanthracycline-Based Chemotherapy on Cognition in Breast Cancer Survivors. JAMA Oncol 2: 185-192.

59. Ng T, Cheung YT, Ng QS, Ho HK, Chan A (2014) Vascular endothelial growth factor inhibitors and cognitive impairment: evidence and controversies. Expert Opin Drug Saf 13: 83-92.

60. Moore HC (2014) An overview of chemotherapy-related cognitive dysfunction, or 'chemobrain'. Oncology (Williston Park) 28: 797-804.

61. Chan RJ, McCarthy AL, Devenish J, Sullivan KA, Chan A (2015) Systematic review of pharmacologic and non- pharmacologic interventions to manage cognitive alterations after chemotherapy for breast cancer. European Journa of Cancer 51: 437-450.

62. Fleishman SB (2004) Treatment of symptom clusters: pain, depression, and fatigue. J Natl Cancer Inst Monogr 119-123.

63. Wang XS, Woodruff JF (2015) Cancer-related and treatment-related fatigue. Gynecol Oncol 136: 446-452.

64. Janelsins MC, Kesler SR, Ahles TA, Morrow GR (2014) Prevalence, mechanisms, and management of cancer-related cognitive impairment. Int Rev Psychiatry 26: 102-113.

65. Galantino ML, Greene L, Daniels L, Dooley B, Muscatello L, et al. (2012) Longitudinal impact of yoga on chemotherapy-related cognitive impairment and quality of life in women with early stage breast cancer: a case series. Explore (NY) 8: 127-135. 